\title{
Exploring the Labyrinths of Resilience: A Concept Analysis
}

\section{Angela Bushati}

MA in "Childhood Studies and Children's Rights" from "Freie Universität Berlin", Germany. Bachelor's degree in "Education" at the "University of Geneva", Switzerland.

\begin{abstract}
This literature review focuses on the concept of resilience seen through an analytical lens as presenting substantial possibilities and an enormous potential mechanism for children experiencing at-risk situations. In many different contexts around the world children face every day different life circumstances and difficulties. Many of these children will experience the "resilience" phenomenon or in other terms they will develop positive adaptation despite all the difficulties and adversities that they encounter. As we are more and more confronted with significant social challenges in many fields and areas of life, also in part posed by the 21st century, the use of the expanding and flourishing knowledge on the concept of resilience could be crucial in advancing and promoting possibilities and well-being for different individuals who are identified as being in a state of risk.
\end{abstract}

Keywords: resilience, concept analysis/definition, research, children and youth at-risk, advantages, limits, possibilities

\section{Introduction}

In the recent decades there has been a growing interest related to the concept of resilience in the field of children's rights and also in other fields. Over time and research on the concept, the term that initially was rather denominated as presenting "invulnerability", in the recent decades has been replaced by the term "resilience". The term portrays or indicates the ability to "bounce back or cope successfully despite substantial adversity" (Rutter, 1987). People surviving and coping positively in extreme life situations experience the phenomenon of resilience. Different researchers from different fields have tried to decorticate the meaning of the concept and have made many efforts to trace resilient attitudes and behaviour among diverse populations of the world. In the resilience research it is assumed that people who experience resilience dispose certain strengths which allow them to succeed or bounce back in high risk situations.

The term resilience appears as useful in various different disciplines such as engineering, economics, business, environment etc. Going back in history, the concept has been stemming initially from engineering sciences/physics where resilience appears as the property of a material capable to go back to its initial state after a force or pressure has occurred. The Latin notion "resilire", translated means to bounce back, to rebound (ISSU, 2015:5). The focus of this paper is "resilience" in terms of human social experience and particularly focusing on children and youth. In the field of humanities the term appears in medicine or early psychiatric literature that for example was analysing children that were "not vulnerable" in 
adverse life situations (Earvolino-Ramirez, 2007). The terms used in psychopathology such as "invulnerable" or "invincible" were replaced later on by the term "resilience" that we use nowadays.

The evolution from these terms to the current use of the term "resilience" came as a result because researchers understood that in different circumstances, individuals presented different degrees of vulnerability or resilience, therefore nothing was absolute. (EarvolinoRamirez, 2007:74) Since that time, the study of children in resilience research has been used to identify possible relationships between risk factors and protectivefactors that lead to resilience, since from several studies (Garmezy, 1981; Rutter, 1979; Werner \& Smith $1982 ; 1989)$ it emerged that the vast majority of children despite experiencing adverse circumstances were demonstrating survival and resilience (Liebel, 2009: 2). For example a lot of researchers are interested in situations such as children living in poverty or children who have experienced war etc. and what are the factors that lead these children to become resilient and have positive outcomes despite facing adversity. The study of resilience has contributed in turning a new page or forming "a new paradigm" in terms of possibilities for capabilities of resistance and empowerment.

According to some researchers resilience can be designated as a "two dimensional construct that implies exposure to adversity and the manifestations of positive adjustment outcomes" and it does not present a personality trait or it is not related to individual attributes. (Luthar \& Cicchetti, 2000:859) Even though many children and young people face different problems or adversities in their life there are many of them who thrive in these difficult situations. This has contributed in giving a huge importance to the concept of "resilience", since it could hold an important key for social changes. If factors that help increase resilience for every child in difficult and at-risk situations such as trauma, family problems, poverty, etc. are being taught or trained, or promoted in some sort of way, the situation for many children could drastically change. Furthermore, the resilience phenomenon or social experience could bring many beneficial outcomes when it comes to the development of children's rights and to the increasing of empowerment and agency of children in the society. Most importantly it could bring positive results and potential impact on well-being and the quality of life. This is why, this concept presents a significant matter and it is important to study what stands behind it.

Although, there has been a lot of theoretical research regarding this construct, it presents a lot of problems and limitations since the concept itself is very variable and multidimensional, very much depending on the context and other variables. Before understanding hidden pathways of resilience, including advantages and limitations or ways of implementing it into social policies, there is a huge need in understanding well its definition and its construct on the first place. A lot of important progress is being madeand has been done so far in the field of resilience but there are still many gaps left open that need to be clarified in order to facilitate a broader understanding of it from research, policy and also practice.

\section{Methodology and research questions}

The objective of this paper is to (i) present a spectrum of the concept of resilience and its construct in terms of attributes, characteristics, etc., (ii) provide an overview of some of the advantages, difficulties and limits related to the conceptualization of this concept, as well as (iii) discuss critically some of the implications for further research. 
The methodology consists in: (i) gathering relevant literature related to the topic of "resilience" by mainly focusing on research from social sciences and particularly by focusing on children and youth, and (ii) discussing it critically in a form of a literature review.

\section{The notion of resilience and the definition of its construct}

The notion of resilience presents multidimensional traits or characteristics and it indicates different specific operational definitions which when it comes to research or theory, itcan be quite difficult to define. Across a lot of literature and research there exists an overall recognition of difficulty in defining the concept. Although it is very difficult to trace a linear definition of the notion, it is more of less acknowledged that it is characterized by two main constructs: (i) adversity and (ii) demonstration of positive outcomes or adaptation. Adversity can be defined as a state presenting a risk and "typically encompasses negative life circumstances that are known to be statistically associated with adjustment difficulties." (Luthar \& Cicchetti, 2000:859) Such risks for example mean experience of trauma, violence, situations of war or massive killings etc. The second important construct of the notion, positive adaptation, includes the adjustment that takes place after being in a state of risk which is manifested in terms ofsocial success or competence, for example good social relations with members of a given society or good emotional state of being.

Overall, according to Masten (1994), the construct of resilience "broadly refers to the class of phenomena involving successful adaptation in the context of significant threats to development" (as cited in Masten \& al., 1999:143). In another definition according to developmental psychology, Garmezy (1985) defined resilience as "the process of capacity for, or outcome of successful adaptation despite challenging or threatening circumstances" (as cited in Windle, 2010:3) Other definitions of the construct can be found in discipline specific dictionaries including juvenile delinquency, medicine, or research in fields such as biology, psychiatry etc. (for further research see Windle 2010:2-5)

According to Luthar et al. (2000), there exists a confusion regarding the conceptualization of resilience viewed mainly as a personal trait and resilience seen rather as a dynamic process. The author argues that resilience rather constitutes a dynamic process and it is not a personality trait. The consideration of resilience as a personality trait can be very detrimental, since it implies that every individual can provide "per se"- by himself and surpass difficulties without any help or services. This is mainly a neo-conservative myth, as Ungar (2005; xvi) also mentions. The author stresses that not every child will be able to develop his/her capacities if there is no actor that helps these children succeed or experience socially the occurrence of resilience. Resilience is not something that depends only from the individual, or residing in him, it is rather a multidimensional, diverse and very complex phenomenon. It is also important to mention that resilience is also not just a process but it concerns different spheres including the individual, the social, the political and other resources within a specific family, community or a given culture.

\section{Main Attributes and protective/risk factors}

The concept of resilience is framed by a huge complexity and the process of defining the attributes brings to a better understanding and clarification of the construct. Since the use of the concept in practice or research requires a good definition of resilience, an in-depth exploration of the most common associated attributes is important and needs to be carefully 
considered. In order for resilience to occur, some factors or attributes are crucial to set up the ability to respond with positive outcomes and diminish the effects of adversity. In order for resilience to occur the presence of risk and protective factors is needed.

Table 1. Protective Factors (by author)

\begin{tabular}{|c|c|c|c|c|c|c|}
\hline Protective factors & Anthony & Benard & Garmezy & Masten & Rutter & Werner \\
\hline Good natured, easy temperament & & $\mathrm{x}$ & & $x$ & $x$ & $x$ \\
\hline Positive relationship & $\mathrm{X}$ & $\mathrm{x}$ & $\mathrm{x}$ & $x$ & $\mathrm{x}$ & $x$ \\
\hline Communicates effectively & & & $\mathrm{X}$ & & $\mathrm{x}$ & \\
\hline Sense of personal worthiness & $\mathrm{x}$ & $\mathrm{X}$ & $\mathrm{X}$ & $\mathrm{x}$ & $\mathrm{x}$ & $\mathrm{x}$ \\
\hline Sense of control over fate & $\mathrm{X}$ & $x$ & $x$ & & & \\
\hline Effective in work, play, love & & & $\mathrm{x}$ & & & \\
\hline Positive social orientation & $\mathrm{x}$ & & $x$ & $\mathrm{x}$ & $\mathrm{x}$ & $\mathrm{x}$ \\
\hline Assertive/asks for help & $\mathrm{x}$ & & $\mathrm{x}$ & & & \\
\hline Above average social intelligence & & & $x$ & & $\mathrm{x}$ & \\
\hline Informal social support network & $\mathrm{x}$ & & $\mathrm{x}$ & & $\mathrm{x}$ & $\mathrm{x}$ \\
\hline Ability to have close relationships & $\mathrm{x}$ & & $\mathrm{x}$ & & $\mathrm{x}$ & \\
\hline Healthy expectations and needs & & & $\mathrm{x}$ & & & $x$ \\
\hline Uses talents to personal advantage & & & $\mathrm{x}$ & $\mathrm{x}$ & $x$ & \\
\hline Delays gratification & $x$ & & $x$ & & $\mathrm{x}$ & $\mathrm{x}$ \\
\hline Internal locus of control & $\mathrm{x}$ & & $\mathrm{X}$ & & $x$ & $\mathrm{x}$ \\
\hline Flexible & $\mathrm{x}$ & & $x$ & & $\mathrm{x}$ & $\mathrm{x}$ \\
\hline Believes in her or his self-efficacy & $\mathrm{x}$ & $x$ & $X$ & $\mathrm{x}$ & $x$ & $x$ \\
\hline Desires to improve & & & $\mathrm{X}$ & & & \\
\hline Interpersonal sensitivity & & & & & $\mathrm{x}$ & $\mathrm{x}$ \\
\hline Problem-solving ability & $\mathrm{x}$ & & $\mathrm{x}$ & & $\mathrm{x}$ & $\mathrm{x}$ \\
\hline Decision-making ability & $\mathrm{x}$ & & $\mathrm{x}$ & & & \\
\hline Future oriented & & & & & $x$ & $\mathrm{x}$ \\
\hline Trust in others/hope for the future & $\mathrm{X}$ & $\mathrm{X}$ & $\mathrm{X}$ & $\mathrm{x}$ & & \\
\hline Sense of humor & $\mathrm{x}$ & $x$ & $\mathrm{x}$ & $\mathrm{x}$ & $\mathrm{x}$ & $\mathrm{x}$ \\
\hline Productive critical thinking skills & $\mathrm{x}$ & & $\mathrm{x}$ & $\mathrm{x}$ & $\mathrm{x}$ & $\mathrm{x}$ \\
\hline Manages range of emotions & $\mathrm{x}$ & & & $\mathrm{x}$ & & \\
\hline Adaptive distancing & & $\mathrm{x}$ & & & & \\
\hline High expectations & $\mathrm{X}$ & $\mathrm{x}$ & $\mathrm{X}$ & $\mathrm{X}$ & $\mathrm{x}$ & $\mathrm{X}$ \\
\hline
\end{tabular}

Fig.1. Protective Factors (by author) (Earvolino-Ramirez, 2007:75)

Vulnerability and protective factors can each originate from multiple levels of influence: the community, family, and the individual (Cicchetti \& Aber, 1986; Cicchetti \& Lynch, 1993; Luthar \& Zigler, 1991; Masten et al., 1990; Werner \& Smith, 1992) as noted in Luthar \& Cicchetti, (2000:860). Risk factors are usually identified as: family factors such as depression, alcohol and drug abuse; family processes such as divorce or separation, loss of one or both parents, family violence; outside factors such as poverty, low income, social conditions of growing up in violent or urban problematic contexts, or in situations resulting of discrimination of minorities or disadvantaged groups. (Liebel,2009:3) On the other side protective factors are often designated as residing between resources of the child, some commonly defined personality characteristics or other attributes of the environment. Earvolino-Ramirez (2007:75) presents some protective factors based on the work of different authors (see Fig.1). Dyer \& McGuiness (1996) argue that "protective factors can be defined as specific attributes or situations that are necessary for the process of resilience to occur" (see Earvolino-Ramirez, 2007:75) However the author mentions that it is important to note that protective factors, since they are contextual, do not qualify as critical attributes since the outcomes can be very different. For the same factors the results can be different for different individuals.

According to different research in resilience some of the defining attributes that appear when the concept occurs are some of the following (as noted in Earvolino- Ramirez, 2007: 76-77): 
Rebounding/Reintegration: bouncing back or reintegrating back to the normal or the familiar.

High expectancy/Self-Determination: sense of expectation or purpose that feeds internal or external goals and self-determination as a sense of perseverance and worth.

Positive Relationships/Social Support: healthy attachment to members of family or other and relationships that provide support and healthy quality.

Flexibility: the ability to adapt with changes, be tolerant and adaptable in different life situations.

Sense of Humor: the ability to have sense of humor about life and oneself.

Self-Esteem/Self-Efficacy: very important in relation to resilience, appearance in different forms and levels.

Another model (see Fig.2) referring to attributes of resilience separates them in three levels such as the individual, the social and the community/society (see Windle, 2010: 6- 7). This model takes in consideration resilience not only on an individual level of attributes. Here resilience is understood as a multidimensional and diverse mechanism.

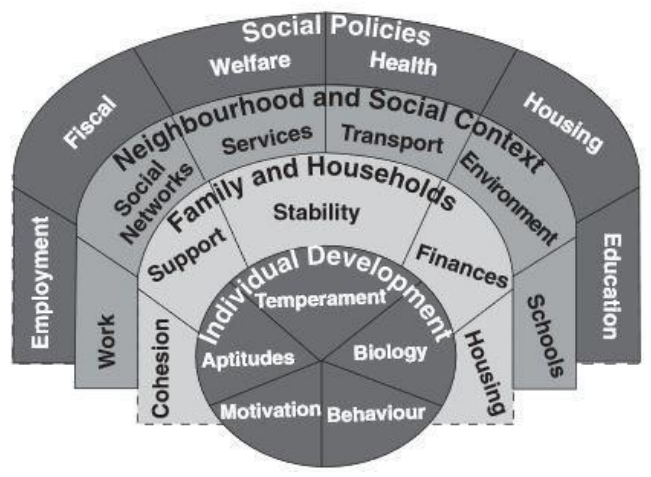

Fig.2. Example of the layers of resources and assets that facilitate resilience (from A. Sacker, personal communication, 2009; adapted from Dahlgren \& Whitehead, 1991 as appearing in Windle, 2010:7)

Other authors such as Ungar et al. (2005:326-327) provide an overview of some attributes starting from the individual (in this case referring to children and youth), interpersonal and beyond the environment including the social and cultural context. 


\section{Individual attributes}

Competence (intellectual, physical), past and present

Self-efficacy, internality

Positive self-concept/self-esteem

Self-awareness or insight

sense of humour/creativity

Positive outlook/optimism/hopefulness

Goals and aspirations/personal mission

Problem-solving ability

Healthy sexual identity

Initiative and planning

Perseverance

Empathy for others

Emotionally expressive

Autonomy and independence or dependency (as appropriate to situation)

Morality

Spirituality

constructive use of time

\section{Interpersonal attributes}

Meaningful relationships with others/social bonding

Maintains a network of school, home, community and peer associations

Emotional management in stressful situations

social competence (understands what motivates others, how to act appropriately, etc)

Assertiveness, resistance to negative and controlling behaviours by others

Capacity to restore self-esteern when threatened by others

interpersonal planning skills

interpersonal problem-solving skills

Evoking personality, engages with others, elicits positive attention

\section{Family attributes}

Parents monitor the children in age appropriate ways

Quality of parenting

Financial resources sufficient to meet family's needs and social expectations

Avoidance of dangerous or threatening family interactions

Family emotional expressiveness

collaborative family problem-solving

Flexibility

Low level of family conflict 


\section{General characteristics of the environment beyond the family (including peers,} school, community)

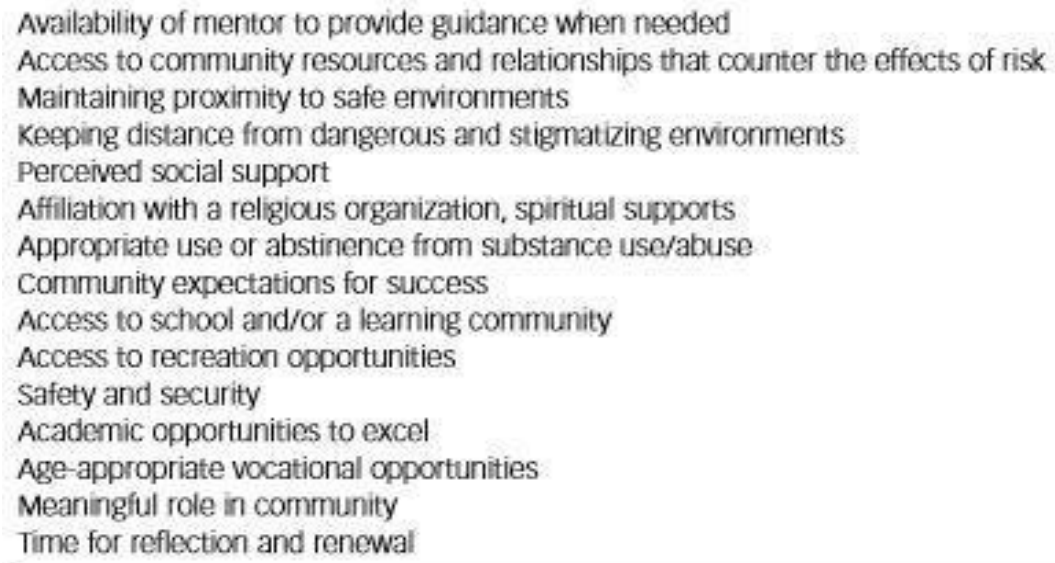

Fig.3. Attributes, environment and social context (Ungar et al., 2005:326-327)

\section{Antecedents and consequences}

Based on the Walker and Avant (2005) method of concept analysis, it appears of extreme importance the need to understand the contexts in which the concept occurs or is applied. Therefore, it is important to also clarify the antecedents and the consequences. Antecedents include the events that happen before the concept itself. On the other hand consequences are the events that proceed after the occurrence of the concept being studied. Adversity is the crucial antecedent before resilience occurs. Without a state ofrisk or adversity, no resilience will appear. After resilience, come consequences which could include outcomes such as ability to cope or coping, tolerance, positive adaptation etc. that can appear in different forms or domains.

\section{Model case and additional cases}

A model case is an illustration of the concept that validates approximately all the defining attributes of the concept and does not include other attributes but only those of the concept mentioned. Additional cases include the contrary case and the related case which serve as a comparison and as an additional method to narrow down defining attributes according to the method of Walker \& Avant (2005). Additional cases provide the concept analysis of examples of what the concept is not, which can be helpful in the process of understanding the essence of the concept. In the case of resilience cases can be interpreted differently according 
to different cultures and contexts, so it is very important to consider this fact. Many examples of cases can be found in different literature such as those in Earvolino-Ramirez (2007: 78-80) or in Dyer \& McGuinness (1996:278-280).

\section{Empirical referents}

The empirical referents state the existence of the phenomenon of resilience. On this we could note that there are many instruments on resilience such as the Resilience Scale for Adults (RSA). This scale is composed by 37 items and it is based on the protective factors defined by different resilience researchers over the years (see Earvolino-Ramirez, 2007). Empirical referents mainly concern the part of how the existence of resilience is measured in practice. The presence of factors such as adversity, the capability of resistance and showing positive outcomes rather than negative ones confirms the occurrence of resilience. There are other approaches that serve to identify resilience such as the variable focused approaches (multivariate statistics) and the person focused approaches (see Windle, 2010:8-10).

\section{Cultural and contextual component of resilience}

Children's development is deeply affected also by the multiple contexts and cultures that they experience. Resilience as well, since it's multidimensional and very diverse, it also depends on the contexts and the cultural component. The opportunities by which individuals are capable to surpass difficulties and situations of adversity are different. It is indeed acknowledged that resilience is relevant in a global perspective but nevertheless, it is also very contextual. The measurements of what it is considered to be a risk or resilience it is very much depending on the cultural perceptions and therefore, there can't be a strictly defined pathway to resilience in a global sense. According to Ungar (2005: xxiv) the definition of risk factors, and specifically the notion of risk, is socially constructed and also culture specific. According to Opp \& Fingerle (2007:15), as mentioned in Liebel (2009:4), regarding the interplay of risk and protective factors the authors mention that it should be understood as an integrated and complex process, where the a-priori made distinctions between risk and protective factors do not always necessarily make sense. According to (Ungar, 2005; Ungar et al. 2007) the social construction of different factors is depending on the context and especially on class, gender, economics or it's culturally specific. Therefore, the study of resilience should refer and include these dimensions, the socio-historical context of children and youth and this applies especially to those who live in socially disadvantages or marginalized groups where resilience takes different forms. The process of defining resilience and implementing approaches that promote resilience sometimes it's tricky since the westernised resilience discourse inflicts the idea of individualism and does not take in consideration the cultural and contextual spaces, but rather conforms disadvantaged children and youth to social norms of the dominant society.

"The resiliency discourse imposes prescribed norms of school and social success upon underprivileged children identified as at risk. The effect is that non-conforming individuals may be pathologized as non-resilient. Emphasis, remains wholly on the individual and thus, individualism is a dominant ideology embedded in the mainstream resiliency discourse" (Martineau, 1999: 11-12 cited in Ungar 2005: XXVI; Liebel, 2009: 6-7)

In addition, as argued in Ungar (2005) the western societies and mentality generally associate more to the dimensions of the individual rather than communal. In many other parts around the world things don't work like this, since individuals are perceived as being part of a 
community or a specific culture which is rather focusing on the "collective" than on the "individual". Therefore, even though the study of attributes or factors on an individual level that lead to resilience could be important, it is also necessary to mention that the dimension of the "communal" or the child/youth experiences related to the collective, shouldn't be underestimated. Instead, they should be valorized. Community resilience (when in confrontations with risks), goes hand in hand with the active action of the subjects. The notion of well-being is not separated from the notion of community or culture. As a result, also the notion of resilience itself cannot be understood on the individual level but it is rather a cultural reflexive and a contextual understanding. It is a "community experience" and an interconnection between the individual and the environment around him.

\section{Advantages and limitations of resilience}

The resilience framework presents many advantages, since it could bring scientific evidence regarding factors that help in the shift or adjustment in high-risk situations. The study of resilience brings explicit guidelines regarding social interventions or policies that may help communities and individuals inside them to experience resilience. "Of central interestare not only adaptational failures (traditionally focused on in research with groups at high risk; Cicchetti, 1993) but also, and more importantly, positive adaptational outcomes and their antecedents (Garmezy, Masten, \& Tellegen, 1984; Luthar \& Zigler, 1991; Masten, et al., 1990)" as noted in Luthar \& Cicchetti (2000: 892). The resilience framework encompasses on strengths, rather than on deficits, which contributes in enhancing the positive aspects or changes in individuals considered at-risk. Some authors mention another important aspect of resilience which is thought to increase the adaptability or the capacity to cope in a specific given environment. "Resilience describes the phenomenon of surviving and thriving in the face of adversity typically predictive of negative outcomes:poverty, family psychopathology, and trauma. Resilience improves conditions affecting an individual's ability to cope." (Kitano \& Lewis, 2005: 200) Furthermore, the resilience approach is very useful when it comes to the process of circumscribing factors, that are powerful when there exists a high-risk situation but they aren't affecting the same when the high-risk situation does not occur. The careful attention also on context-specific vulnerability and protective factors gives the approach a great importance on the macro and micro level. The UN Convention on the Rights of the Child (CRC) puts a great emphasis on the child's well-being. As a result, the protection of children (considered as being in a high-risk situation who face adversity) has become a crucial priority of interventions and policies in an international level. Since the conception of well-being is also contextual, there are many difficulties in implementing the CRCs mechanisms. Therefore, the protection of children is still all over the world very uncertain. New approaches, such as the resilience approach, provide exploration of dimensions related to children's experiences, which are inseparable from their contexts.

On another level, beside many advantages that resilience presents, there are also difficulties regarding the terminology of this concept due to its variability. There are some problems related to the methodology and the non-adequate application of scientific standards. Different researchers use a huge variety of methodology and sometimes the application of theory in the field of research is not made accurately. Another concern refers to the use of another term, "hardiness", which sometimes is often misinterpreted or misunderstood as resilience from people. The term "hardiness", is described as "robust or able to withstand adverse conditions" according to the Merriam-Webster Dictionary (2002) as noted in Earvolino-Ramirez (2010:80) and it is rather a personality trait, which resilience is not. 
Resilience leads to positive adaptive outcome and hardiness doesn't necessarily bring a positive outcome, it's more a mechanism of self-endurance. One of the major problems in resilience research can be the misuse of the term for implying a personality trait, which can be a very prodigious problem. Such consideration would imply that children possess this trait that enables them to endure and succeed in situations of risk, which indirectly puts the blame on the individual level if this trait is missing. This could serve also as a justification from stakeholders in order not to intervene or offer support for children who face high-risk conditions. According to Doll \&Lyon (1998:360) this mentality puts children in a state of being "responsible for forging their own Horatio Alger pathway through risk and toward success".

A big limitation in the study of resilience is the process of defining a situation at-risk, an individual being at-risk, since the assumption can be biased. Resilience outcomes are also linked to normative judgements. Furthermore, there is a striking difference between the research priorities in the global north and south (Liebel, 2011:13). In the northern part, the focus is mostly on the individual level and in the south there is a focus in greater social events such as wars, poverty etc. For example, Werner (1993) argues that there is a limitation and danger in the process of defining outcomes because sometimes it could be generalized based on principles of a specific culture. Risk factors and the conceptualizations of resilience are very variable and diverse according to normative judgements of a specific context and the subjective view of the researcher.

Another main issue, which leads to limited interpretation, is also the fact that most of the research on childhood experience is mainly based on adult perspectives on childhood and children. Constructions of childhood and adulthood and their dichotomy interfere on the characterization of what is considered to be a situation of resilience, what is a risk etc. According to Boyden \& Mann (2005: 15), there is no accurate information on children's own perspectives and since they don't share the same perspectives, this can be quite problematic. As cited in Liebel (2009:5), Boyden and Mann (2005:15) argue that "the privileging of adult perceptions over children's experiences has sometimes meant that, in practice, resilience is conceived of more as the absence of pathology rather than the presence of personal agency in children". The majority of children around the world are relying on the parental support and care and often adults are the ones who decide about their children and their well-being.

According to the CRC, adults are entitled and are morally obliged to protect and take care of children. This includes protection against all situations of risk and also adversity. Nevertheless, it is a fact that in western societies, children are being overprotected andtheir agency is often restricted. "Modern societies have generally tried to defend against risks at all costs meaning that we are less likely to accept fate as an explanatory factor for peril"(O'Hare et al. 2015:3). The fact that children are conceptualized from adults as in need to be protected, indirectly harms experiencing resilience and coping. This poses a paradox as it does not mean that support is not needed, but it rather tends to articulate that children should be considered in research as active social actors, rather as adult future projects or products of intervention etc. Boyden \& Mann (2005:19) suggest that "supporting children in situations of adversity requires the perspective not just that children need special protection but that they have valid insights into their well-being, valid solutions to their problems, and a valid role in implementing those solutions." 


\section{Implications for further research}

Resilience research may hold significant potential for social changes especially regarding social policies or other, since its main concern are factors that adjust the effects of difficult and at-risk life conditions.

The exploration of resilience is central and consequential in the process of instrument development and application. The study of resilience, even though it has been known for its development in the recent decades, it is spread in different fields of study and has a relatively sufficient background regarding theory and research. The attention and the commitment for future research should be focused on exploring new ways on how to apply resilience on interventions that help children of different social backgrounds or different contexts. Of importance is also the development of interventions that are not related to age. The application of resilience research can bring substantial outcomes for many fields by promoting positive outcomes, increased adaptability and ability to cope.

According to Windle (2010: 13) most of the resilience research refers to children and adolescents. The author states that regarding resilience in adulthood, not much has been studies and less is known. Therefore more research is needed regarding the follow up ofprotective factors within the entire life course and according to age. In addition, it might result interesting to research whether factors that bring to adult resilience could be similar or not to those represented in childhood or adolescents. The study of resilience could be deepened also by the contributions from other fields such as neuroscience, biology etc. which would help examine factors from different perspectives. Multi- disciplinary approaches could help discover other underlying factors or fluctuations of resilience. Research on dynamics and mechanisms of adversity, interventions or other protective elements have not been widely conducted, even though it is very important (Sandler, 2001). Further research and importance should be also focused on psychological resilience vs. improving resources (such as social support) or the study of the dynamics between these levels (Windle, 2010: 14). Luthar \& Cicchetti (2000) argue in favor of the need to increase the interface between science and practice in the development of interventions and evaluation mechanisms.

\section{Discussion}

The literature review shows that the available theoretical background of the concept construct is very broad and also very variable which also poses some limitations in the process of evaluation. In many different fields or even diverse contexts, resilience is approached or conceptualized in many ways. According to Ungar (2005) "a broad developmental perspective on resilience that can fully account for how children become resilient in multiple contexts across cultures has yet to be fully articulated." (xvii)

The study of resilience, including risk and protective factors or other components may be very beneficial to the realization of a change in terms of well-being, positive adaptation to difficult life conditions and a source of empowerment for many children facing adversity around the world. The process of definition of different factors needs to take into account the contextual components that take part in the construction of protective/risk factors such as gender, social classes, cultural specificities and normative judgements. Risk factors and community resilience can only be understood if theinterpretations and the use of the employed terms 
is context specific. As a result, they can't be generalized and a holistic approach is strongly envisaged.

From the resilience perspective, adversity and risks are not considered in isolation, but they explore children's opportunities to learn from them and become stronger (Liebel, 2011:10). This could be particularly important in regard to children who face different vulnerabilities, social exclusion, discrimination etc. due to different social situations, since their adaptability and flexibility may be activated in a cul-de-sac situation. The resilience paradigm opens up several possibilities also regarding new ways of understanding and analyzing for example children in street situations, as noted also by Liebel (2011: 3). In addition, children are seen as acting subjects in their own interpretation of the world and their specific competencies have to be taken seriously. For example, children are not only considered in terms of their relationship with the street but also their life story and their diverse social relations come into view. This conceptualization opens up important opportunities that could lead to relevant steps in the realization of children's rights around the world.

Resilience, a notion which emerges as a possible alternative solution to many problematics that the world is facing, besides its many positive constituents, exposes a paradox of our times. Matthias Horx (ISSU, 2015:6) notes on this perspective that resilience will replace the concept of sustainability, behind which stands an old harmony- illusion that revolutionary systems move always on the borders of chaos. The normative dimension of sustainability suggests to shape the conditions that minimize the dangers and adverse life circumstances. In this logic, the staple is no longer to make efforts to correct or minimize difficult and adverse conditions, but to lead to a process of adaptation, due to lack of alternatives, as part of a progressive and destructive process. Here, resilience could apprehend a pure commercial interest. For example, "insurance regimes could reinforce exposure and vulnerability through underwriting a return to the 'status-quo' rather than enabling adaptive behaviour" (O'Hare et al. 2015). The paradox stands in the fact that those circumstances that are stabilized or "insured", are exactly those precarious conditions that ignite resilience itself.

\section{Conclusion}

Resilience remains nowadays a widely used term, but due to its fluctuating nature, it is quite hard to measure or define it properly. Researchers acknowledge that resilience is relevant in a global perspective but nevertheless, it is also very contextual. Meanwhile, it is imperative to comprehend that the resilience concept needs to be handled with adequacy and conscientiousness, since it could be easily mishandled and it could serve as a strong political tool, as a means of governing the uncertainty, which could possibly turn to a scientific utopia of making the unsafe controllable. If transposed on the individual level, many stakeholders could also take advantage of it to blame the individuals for their own misery or difficulties in life. Therefore, it is vital to comprehend resilience with the help of a multidimensional approach and apply it in a genuine way, as a definition that arises on the basis of social experience and in cooperation with others.

\section{References}

[1] Boyden, Jo \& Gillian Mann (2005): Children's Risk, Resilience, and Coping in Extreme Situa-tions, in: Ungar (2005a), 3-25. 
[2] Doll B, Lyon MA. (1998). Risk and resilience: Implications for the delivery of educational and mental health services in schools. School Psychology Review, 27:348-363.

[3] Dyer, J., \& McGuinness, T. (1996). Resilience: analysis of a concept. Archives of Psychiatric Nursing, 10:276-282.

[4] Earvolino-Ramirez, M. (2007). Resilience: A concept analysis. Nursing Forum, 42:7382.

[5] Garmezy, N. (1985). Stress resistant children: The search for protective factors. In Stevenson, JE. (ed). Recent Research in Developmental Psychology. J Child Psychol Psychiat (book supplement no. 4).

[6] Kitano, M. \& Lewis, R. (2005). Resilience and coping: Implications for gifted children and youth at risk. Roeper Review, 27:200-205.

[7] Liebel, M. (2011). Eigensinnige Wege-Kinder in Straßensituationen. InM. Zander (Hrsg.): Handbuch Resilienzförderung (532-554). Wiesbaden: VS.

[8] Liebel, M. (2009). Kinderrechte - aus Kindersicht. Wie Kinder weltweit zu ihrem Recht kommen. Berlin/Münster: LIT, 47-62.

[9] Luthar, S. S. \& Cicchetti D. (2000). The construct of resilience: implications for interventions and social policies. Dev. Psychopathol.12:857-85.

[10] Luthar, S. S., Cicchetti, D. \& Becker, B. (2000). The construct of resilience: A critical evaluation and guidelines for future work. Child Development, 71: 543-562.

[11] Masten, AS., Hubbard JJ, Gest SD, Tellegen A, Garmezy N, Ramirez M. (1999). Adaptation in the context of adversity: Pathways to resilience and maladaptation from childhood to late adolescence. Development and Psychopathology. 11:143-169.

[12] O'Hare, P. White, I \& Connelly, A. (2015). Resilience and the 'business as usual' paradox.

[13] Environment and Planning C: Government and Policy. 0(0):1-19.

[14] Rutter, M. (1987). Psychosocial resilience and protective factors. American Journal of Orthopsychiatry, 57: 316-331.

[15] Sandler, I. (2001). Quality and ecology of adversity as common mechanisms of risk and resilience. Am J Community Psychol, 29:19-6.

[16] Ungar, M. (Ed.). (2005). Handbook of working with children and youth: Pathways to resilience across cultures and contexts. Thousand Oaks, CA: Sage

[17] Ungar, M., Dumond, C. and McDonald, W. 2005. Risk, resilience and outdoor programmes for at-risk children. Journal of Social Work, 5(3): 319-338.

[18] Walker, L. \& Avant, K. (2005). Strategies for theory construction in nursing (4th ed.). Upper Saddle River, NJ: Pearson Prentice Hall.

[19] Wemer, E.E. (1993). Risk, resilience, and recovery: Perspective from the Kauai longitudinal study. Development and Psychopathology, 5:503-515.

[20] Windle, G. (2010). The Resilience Network: What is resilience? A systematic review and concept analysis. Reviews in Clinical Gerontology. 21:1-18.

\section{Other Resources}

Convention on the Rights of the Child

http://www.ohchr.org/en/professionalinterest/pages/crc.aspx

Consulted on: 15.08 .2015 
ISSU, Medico international - rundschreiben 02/15 Paradox der Resilienz. Nepal: Selbsthilfe nach dem Beben Mittelmeer: Seenotrettung reicht nicht Resilienz-Debatte: Fit fürs Chaos?

http://issuu.com/medico international/docs/rs 15-02 Consulted on 10.09.2015

Figures

Figure 1. Protective Factors (by author). Adapted from "Resilience: A concept analysis", by M. Earvolino-Ramirez (2007), Nursing Forum, 42:75.

Figure 2. Example of the layers of resources and assets that facilitate resilience. Adapted from "The Resilience Network: What is resilience? A systematic review and concept analysis", by G. Windle (2010), Reviews in Clinical Gerontology. 21:7.

Figure 3. Attributes, environment and social context. Adapted from "Risk, resilience and outdoor programmes for at-risk children", by Ungar, M., Dumond, C. and McDonald, W. 2005, Journal of Social Work, 5(3): 326-327. 